\title{
Blockage of AMPK-ULK1 Pathway Mediated Autophagy Promotes Cell Apoptosis to Increase Doxorubicin Sensitivity in Breast Cancer (BC) Cells: An in vitro Study
}

Libo Yu

Harbin Medical University Cancer Hospital

Qingtao Shi

Harbin Medical University Cancer Hospital

Yan Jin

Harbin Medical University Cancer Hospital

Zhixin Liu

Harbin Medical University Cancer Hospital

Jiaxin Li

Harbin Medical University Cancer Hospital

Wenzhou Sun ( $\nabla$ sunwenzhou001111@163.com )

Harbin Medical University Cancer Hospital https://orcid.org/0000-0002-4251-1154

Primary research

Keywords: breast cancer, autophagy, chemoresistance, doxorubicin, AMPK-ULK1 signal pathway

Posted Date: July 30th, 2020

DOI: https://doi.org/10.21203/rs.3.rs-49604/v1

License: (c) (i) This work is licensed under a Creative Commons Attribution 4.0 International License.

Read Full License 


\section{Abstract}

Background: Activation of autophagy flux contributed to resistance of breast cancer (BC) cells to current chemotherapeutic drugs, which seriously limited their therapeutic efficacy and facilitated BC recurrence in clinic. However, the detailed mechanisms are still not fully understood. In the present study, we identified that inactivation of AMPK-ULK1 signaling cascade mediated protective autophagy sensitized BC cells to doxorubicin in vitro.

Methods: Cell counting kit-8 (CCK-8) assay and colony formation assay were performed to evaluate cell proliferation abilities. Trypan blue staining assay was used to examine cell viability, and Annexin VFITC/PI double staining method was conducted to determine cell apoptosis. The autophagosomes in BC cells were observed and photographed by electronic microscope (EM). Western Blot analysis was employed to examine genes expressions at protein levels.

Results: The parental doxorubicin-sensitive BC (DS-BC) cells were exposed to increasing concentrations of doxorubicin to establish doxorubicin-resistant BC (DR-BC) cells, and the DR-BC cells were much more resistant to high-dose doxorubicin stimulation compared to the DS-BC cells $(P<0.05)$. Interestingly, highdose doxorubicin specifically increased LC3B-II/I ratio, promoted autophagosomes formation and decreased p62 expression levels to facilitate autophagy in DR-BC cells, instead of DS-BC cells $(P<0.05)$, and the autophagy inhibitor 3-methyladenine (3-MA) enhanced the cytotoxic effects of high-dose doxorubicin on DR-BC cells $(P<0.05)$. In addition, we proved that high-dose doxorubicin triggered protective autophagy in DR-BC cells by activating AMPK-ULK1 pathway. Functionally, high-dose doxorubicin increased the expression levels of phosphorylated AMPK (p-AMPK) and ULK1 ( $p$-ULK1) to activate AMPK-ULK1 pathway in DR-BC cells $(P<0.05)$, and the inhibitors for AMPK (compound C) and ULK1 (SBI-0206965) blocked autophagy to promote cell death and slow down cell growth in DR-BC cells treated with high-dose doxorubicin $(P<0.05)$.

Conclusions: Collectively, our in vitro data indicated that blockage of AMPK-ULK1 signaling cascade mediated protective autophagy might be a promising strategy to increase doxorubicin sensitivity for BC treatment.

\section{Background}

Although great advances had been reached for breast cancer (BC) treatment, BC still brought huge health burden to women worldwide as the results of high frequency of metastasis and chemo-resistance, and searching for strategies to improve the therapeutic efficacy for current chemical drugs might help to cure $\mathrm{BC}$ in clinic [1,2]. Among all the chemotherapeutic drugs, doxorubicin (Dox) was commonly used for cancer treatment, including non-small cell lung cancer (NSCLC) [3], ovarian cancer [4], hepatocellular carcinoma (HCC) [5], BC [6-8], etc.. However, Dox-resistance had became an insurmountable obstacle for cancer treatment $[9,10]$, which made this drug "ineffectiveness" for BC treatment, resulting in worse prognosis and recurrence in $\mathrm{BC}$ patients [11]. To solve this problem, the combination treatment had been 
developed by researchers to increase Dox-sensitivity [7, 12]. For example, Ji Wu et al. reported that inhibition of N-acetyltransferase 10 reversed Dox-resistance in BC [12], and Yingze Wei et al. verified that Nicotinamide overcame Dox-resistance in BC [7]. Therefore, this study aimed to develop a novel strategy to improve Dox-sensitivity in BC.

Autophagy is a evolutionarily conserved process for cells in defense of environmental stress, which degraded and reused the destructed cellular constitutes and cytoplasmic organelles to protect cells from death $[13,14]$. Recent data agreed that LC3 (microtubule-associated protein light chain 3 ) and p62 could be used to test autophagy activities, and increase of LC3-II (membrane bound)/LC3-I (cytosolic section), and degradation of p62 indicated activation of autophagy flux $[15,16]$. Interestingly, autophagy was closely associated with chemo-resistance in cancer treatment, and inhibition of autophagy had been proved to be effective strategy to increase drug-sensitivity for multiple drugs, including cisplatin [17], 5-FU [18], Dox [19, 20], etc., and Zhenyu Liu et al. [21] and Lu Liang et al. [22] agreed that targeting autophagy was novel to enhance Dox-sensitivity for BC treatment. Nevertheless, the detailed mechanisms are still not fully understood. To solve this issue, the inhibitor for autophagy, 3-methyladenine (3-MA) [23], was used in this study, and we validated that 3-MA aggravated the inhibiting effects of Dox on cell proliferation in Dox-resistant BC (DR-BC) cells, which rendered the possibility that autophagy might play a protective role to enhance Dox-resistance under high-dose Dox stimulation in BC cells.

AMP-activated protein kinase (AMPK)-Unc-51-like kinase 1 (ULK1) signaling cascade was crucial for autophagy initiation [24-26], and activation of AMPK-ULK1 pathway triggered protective autophagy to promote cell survival under environmental stress, such as oxidative stress [27] and cigarette smoke exposure [28]. Aside from that, recent data indicated that AMPK-ULK1 pathway mediated autophagy involved in regulating drug resistance during cancer treatment, including cisplatin [29,30] and Dox [21]. Specifically, Guihua Duan et al. [30], Lixiao Che et al. [29] and Zhenyu Liu et al. [21] reported that induction of AMPK-ULK1 pathway mediated autophagy contributed to cisplatin- and Dox- resistance in ovarian cancer and $\mathrm{BC}$, respectively. The above information enlightened us to speculate that AMPK-ULK1 pathway mediated protective autophagy might be the reason of Dox-resistance in BC. To verify this hypothesis, the inhibitors for AMPK (compound C) and ULK1 (SBI-0206965) [31] were used to treat BC cells, and we noticed that both compound C and SBI-0206965 increased Dox-sensitivity in BC cells.

Based on the existed information, by conducting in vitro experiments, we managed to investigate whether autophagy blockage was effective to increase Dox-sensitivity in BC cells. Also, further experiments were performed to uncover the underlying mechanisms, and explored the role of AMPK-ULK1 pathway mediated protective autophagy in regulating Dox-resistance in BC cells. The present study will provide evidences to support that inhibition of AMPK-ULK1 pathway mediated protective autophagy is a novel strategy to improve Dox-sensitivity in BC.

\section{Methods}

\section{Cell culture and treatment}


The human breast cancer (BC) cell lines (MDA-MB-231 and MCF-7) were purchased from American Type Culture Collection (ATCC, USA), and cultured in Dulbecco's modified Eagle's medium (Invitrogen, USA) containing $10 \%$ fetal bovine serum (FBS, Hyclone, USA), $100 \mathrm{U} / \mathrm{ml}$ penicillin and $100 \mu \mathrm{g} / \mathrm{ml}$ streptomycin in an incubator with $5 \% \mathrm{CO}_{2}$ humidified atmosphere at $37{ }^{\circ} \mathrm{C}$. The cells were harvested for further experiments until the cell confluency reached about $70-80 \%$, and were subjected to increasing concentrations $(0.01-1 \mu \mathrm{g} / \mathrm{ml})$ of doxorubicin (Sigma, USA) in a step-wise manner over a period of 6 months to generate doxorubicin-resistant $B C$ (DR-BC) cells based on the experimental protocol provided by the previous publication $[32,33]$. To sustain the doxorubicin-resistant phenotypes, the DR-BC cells were cultured in the DMEM medium supplemented with $0.01 \mu \mathrm{g} / \mathrm{ml}$ doxorubicin. After that, the DR-BC cells were pre-treated with $10 \mu \mathrm{mol} / \mathrm{L}$ of AMPK inhibitor compound C, $6 \mu \mathrm{mol} / \mathrm{L}$ ULK1 inhibitor SBI-0206965 and $12 \mathrm{mmol} / \mathrm{L}$ of autophagy inhibitor 3-methyladenine (3-MA) for $24 \mathrm{~h}$, and subsequently stimulated with high-dose doxorubicin $(20 \mu \mathrm{g} / \mathrm{ml})$ for $0 \mathrm{~h}, 24 \mathrm{~h}, 48 \mathrm{~h}$ and $72 \mathrm{~h}$.

\section{Western Blot analysis}

The total protein was extracted from the BC cells by using a commercial RIPA lysis buffer, and the following Western Blot analysis was conducted to examine the protein levels of the involved genes, and all the detailed experimental procedures had been well documented in the previous work [32, 33]. The primary antibodies were listed as follows: Cyclin D1 (1:1500, Abcam, UK), CDK2 (1:2000, Abcam, UK), cleaved caspase-3 (1:2000, Abcam, UK), Bax (1:2000, Abcam, UK), $\beta$-actin (1:3000, Abcam, UK), LC3B (1:1000, Abcam, UK) and p62 (1:1000, Abcam, UK).

\section{Cell counting kit-8 (CCK-8) assay}

The BC cells were subjected to different treatments, and cell proliferation was evaluated by using the commercial CCK-8 assay kit purchased from Abmole (USA) according to the manufacturer's protocol. Briefly, the cells were seeded into the 96-well plates, and cultured in the incubator for $0 \mathrm{~h}, 24 \mathrm{~h}, 48 \mathrm{~h}$ and $72 \mathrm{~h}$, respectively. Next, the cells were incubated with $20 \mu \mathrm{l}$ CCK-8 reaction solution, and the optical density values were examined to reflect relative cell proliferation abilities.

\section{Colony formation assay}

The BC cells were harvested and administered with different stimulation, after that, the cells were cultured in the 6-well plates at the concentration of 500 cells per well. After 14 days culture in the incubator, the cells were stained with $0.4 \%$ crystal violet for visualization. Next, the cells were observed and photographed under light microscope, and the cell colonies containing at least 10 cells were counted.

\section{Trypan blue staining assay}


The $\mathrm{BC}$ cells were collected, and cell viability was determined by using the trypan blue staining method. The cells were stained with the trypan blue staining solution for $20 \mathrm{~min}$ at $37^{\circ} \mathrm{C}$, and the dead blue cells were observed, photographed and counted under light microscope. The formula for calculating cell viability was shown as follows: cell viability $(\%)=($ total cell numbers - dead blue cell numbers)/total cell numbers $* 100 \%$.

\section{Measurement of cell apoptosis}

The commercial apoptosis detection kit (BD Bioscience, USA) was purchased to measure cell apoptosis ratio in $\mathrm{BC}$ cells with differential stimulation, and the detailed experimental procedures were recorded in the manufacturer's instruction. In brief, the cells were double stained with Annexin V-FITC and PI for 30 min at room temperature without light exposure, and a flow cytometer (ThermoFisher Scientific, USA) was used to examine and count the numbers for apoptotic cells.

\section{Observation of autophagosomes by electronic microscope (EM)}

The BC cells, including DS-BC and DR-BC cells, were fixed with $0.1 \mathrm{M}$ sodium cacodylate-buffered $2 \%$ (wt/vol) glutaraldehyde at $4{ }^{\circ} \mathrm{C}$ overnight, and pelleted in agarose, rinsed in distalled water and stored in $70 \%$ ethanol. After that, the cells were dehydrated and stained with $2 \%$ aqueous uranyl acetate for 5 min, and Reynolds lead citrate for 3 min at room temperature. Finally, the cells were observed and photographed by an electronic microscope (EM) at $80 \mathrm{kV}$.

\section{Statistical analysis}

All the data were presented as Means \pm Standard Deviation (SD), and the data were analyzed and visualized by suing the SPSS 18.0 software and GraphPad Prism 7.0 software. Specifically, the comparisons of means from two groups were conducted by using the unpaired Student's t-test, and the means from multiple groups were compared by using the one-way ANOVA analysis. $P<0.05$ was regarded as statistical significance, and marked by " " in the Figures. Each experiment repeated at least 3 times.

\section{Results}

\section{Induction of doxorubicin-resistant BC (DR-BC) cells from their corresponding parental doxorubicin-sensitive BC (DS- BC) cells}


According to the previous work [32], the parental DS-BC cells (MDA-MB-231 and MCF-7) were subjected to continuous low-dose doxorubicin stimulation, starting from $0.01 \mu \mathrm{g} / \mathrm{ml}$ and terminating at $1 \mu \mathrm{g} / \mathrm{ml}$, to induct DR-BC cells (DR-MDA-MB-231 and DR-MCF-7). After that, based on our preliminary experiments (data not shown), the above cells were exposed to high-dose doxorubicin $(20 \mu \mathrm{g} / \mathrm{ml})$ stimulation for $0 \mathrm{~h}$, $24 \mathrm{~h}, 48 \mathrm{~h}$ and $72 \mathrm{~h}$, respectively, and the results in Fig. 1 showed that we had successfully established DR-BC cells in vitro. Mechanistically, the CCK-8 assay (Fig. 1A-D) and colony formation assay (Fig. 1E, F) results showed that high-dose doxorubicin significantly inhibited cell proliferation and colony formation abilities in DS-BC cells, instead of their corresponding DR-BC cells. In addition, high-dose doxorubicin stimulation decreased cyclin D1 and CDK2 expression levels to induce cell-cycle arrest in DS-BC cells, but had little effects on the above proteins in DR-BC cells (Fig. 1I-N). Consistently, the trypan blue staining (Fig. 1G, H) and Annexin V-FITC/PI double staining assay (Fig. 10-Q) results provided evidences to support that high-dose doxorubicin specifically inhibited cell viability and promoted cell apoptosis in DS$\mathrm{BC}$ cells compared to the DR-BC cells, which were also validated by the following Western Blot analysis for the pro-apoptosis associated proteins (Fig. 1R-W). Specifically, the results showed that high-dose doxorubicin increased the expression levels of cleaved caspase-3 and Bax in DS-BC cells, but did not influence their expression status in DR-BC cells (Fig. 1R-W). The above results indicated that DR-BC cells were much more resistant to high-dose doxorubicin stimulation in contrast with their corresponding parental DS-BC cells.

\section{High-dose doxorubicin stimulation specifically triggered autophagy in DR-BC cells}

Previous work suggested that induction of autophagy was crucial for doxorubicin resistance in BC [3436], however, the detailed mechanisms are still controversial. To investigate this issue, the DS-BC and DR$B C$ cells were exposed to high-dose doxorubicin $(20 \mu \mathrm{g} / \mathrm{ml})$ stimulation for $48 \mathrm{~h}$. As shown in Fig. 2, we proved that autophagy flux was specifically activated by high-dose doxorubicin in DR-BC cells, instead of DS-BC cells. Functionally, the Western Blot analysis results showed that high-dose doxorubicin significantly increased LC3B-II/I ratio and decreased p62 expression levels in DR-BC cells, but did not altered their expression patterns in DS-BC cells (Fig. 2A-F). In addition, the transmission electron microscopy images indicated that high-dose doxorubicin specifically induced autophagosomes formation in DR-BC, instead of their parental DS-BC cells (Fig. 2G, H). Furthermore, the DR-BC cells were pretreated with $12 \mathrm{mmol} / \mathrm{L}$ of autophagy inhibitor 3-methyladenine (3-MA) for $24 \mathrm{~h}$, and we found that the effects of high-dose doxorubicin stimulation on LC3B-II/I ratio and p62 expressions in DR-BC cells were abolished by co-treating cells with 3-MA (Fig. 2I-L), indicating that 3-MA abrogated high-dose doxorubicin-induced autophagy in DR-BC cells.

\section{Inhibition of autophagy aggravated high-dose doxorubicin induced cell death in DR-BC cells}


We next investigated whether blocking autophagy increased doxorubicin sensitivity in DR-BC cells. To achieve this, the DR-BC cells were pretreated with $3-\mathrm{MA}(12 \mathrm{mmol} / \mathrm{L}, 24 \mathrm{~h})$ and subsequently stimulated with high-dose doxorubicin $(20 \mu \mathrm{g} / \mathrm{ml}, 48 \mathrm{~h})$. The CCK-8 assay (Fig. 3A, B) and colony formation assay (Fig. 3C, D) results showed that 3-MA aggravated the inhibiting effects of high-dose doxorubicin on cell proliferation and colony formation abilities in DR-BC cells, however, 3-MA alone had little effects on the above cell functions (Fig. 3A-D). In addition, the trypan blue staining assay results showed that 3-MA inhibited cell viability in DR-BC cells treated with high-dose doxorubicin (Fig. 3E), which were supported by the following Annexin V-FITC/PI double staining assay results and showed that 3-MA increased apoptosis ratio in high-dose doxorubicin stimulated DR-BC cells (Fig. 3F, G). Similarly, 3-MA alone had little effects of cell death in DR-BC cells (Fig. 3F, G). Furthermore, the proliferation and apoptosis associated proteins were also determined by using the Western Blot analysis, and the results showed that high-dose doxorubicin combined with 3-MA significantly inhibited cyclin D1 and CDK2, and upregulated cleaved caspase-3 and Bax in DR-BC cells (Fig. $3 \mathrm{H}-\mathrm{K}$ ). The above results suggested that inactivation of autophagy by 3-MA enhanced the cytotoxic effects of high-dose doxorubicin on DR-BC cells.

\section{Activation of AMPK-ULK1 signal pathway contributed to high-dose doxorubicin induced autophagy in DR-BC cells}

Further experiments were conducted to explore the underlying mechanisms of high-dose doxorubicin induced autophagy in DR-BC cells. Based on the previous studies [26, 37], the AMPK-ULK1 signaling cascade was selected for further analysis. As expected, the Western Blot analysis results showed that high-dose doxorubicin specifically increased the expression levels of phosphorylated AMPK (p-AMPK), pULK1 (555) and p-ULK1 (757) to activate AMPK-ULK1 signal pathway in DR-BC cells, however, high-dose doxorubicin did not influenced AMPK-ULK1 activation in DS-BC cells (Fig. 4A, B), which enlightened us that AMPK-ULK1 signal pathway might be crucial for high-dose doxorubicin induced autophagy in DR-BC cells. To investigate the above speculation, the DR-BC cells were pretreated with $10 \mu \mathrm{mol} / \mathrm{L}$ of AMPK inhibitor compound $\mathrm{C}$ and $6 \mu \mathrm{mol} / \mathrm{L}$ ULK1 inhibitor SBI-0206965 for $24 \mathrm{~h}$ to inactivate AMPK-ULK1 signaling cascade, and the results showed that compound $C$ decreased $\mathrm{p}$-AMPK expression levels (Fig. 4C, D) and SBI-0206965 inhibited p-ULK1 (555) and p-ULK1 (757) expressions (Fig. 4E, F) in highdose doxorubicin treated DR-BC cells. Furthermore, we found that the regulating effects of high-dose doxorubicin on LC3B-II/I ratio and p62 expressions in DR-BC cells were all abrogated by co-treating cells with compound C and SBI-0206965 (Fig. 4G, H), indicating that high-dose doxorubicin activated autophagy flux in DR-BC cells by activating AMPK-ULK1 signal pathway.

\section{Abrogation of AMPK-ULK1 signal pathway mediated protective autophagy increased doxorubicin sensitivity in DR-BC cells}


Given the fact that high-dose doxorubicin activated AMPK-ULK1 signaling cascade to promote autophagy in DR-BC cells, and blockage of autophagy enhanced cytotoxic effects of high-dose doxorubicin on DRBC cells. As indicated in Fig. 5, we validated that inactivation of AMPK-ULK1 pathway increased doxorubicin sensitivity in DR-BC cells. Specifically, as mentioned before, the DR-BC cells were preadministered with compound C (10 $\mu \mathrm{mol} / \mathrm{L})$ and SBI-0206965 $(6 \mu \mathrm{mol} / \mathrm{L})$ for $24 \mathrm{~h}$ to inactivate AMPKULK1 pathway, and subsequently stimulated with high-dose doxorubicin $(20 \mu \mathrm{g} / \mathrm{ml})$ for $48 \mathrm{~h}$. As expected, the CCK-8 (Fig. 5A, B) results showed that both compound C and SBI-0206965 significantly inhibited cell growth in high-dose doxorubicin treated DR-BC cells. Consistently, by conducting the trypan blue staining assay, we found that the inhibitors for AMPK-ULK1 pathway also aggravated the inhibiting effects of doxorubicin on cell viability in DR-BC cells (Fig. 5C). Also, blockage of AMPK-ULK1 pathway also triggered apoptotic cell death in DR-BC cells, and both compound C and SBI-0206965 induced cell apoptosis in doxorubicin treated DR-BC cells (Fig. 5D, E).

\section{Discussion}

Doxorubicin (Dox) was commonly used for breast cancer (BC) treatment in clinic [6-8], however, the therapeutic efficacy of this chemical drug was seriously limited as the results of drug resistance $[9,10]$, resulting in worse prognosis and recurrence in $\mathrm{BC}$ patients [11]. Recent data indicated that combination treatment strategies might be novel to increase Dox-sensitivity for $B C$ treatment $[7,12]$, hence, the present study managed to investigate this issue in vitro. To achieve this, based on the information from the previous publications $[32,33]$ and our preliminary experiments (data not shown), the Dox-resistant BC (DR-BC) cells were inducted from their parental Dox-sensitive BC (DS-BC) cells through long-term continuous Dox exposure method in a step-wise manner. After that, both the DR-BC and DS-BC cells were stimulated by high-dose Dox, and the results showed that Dox downregulated cyclin D1 and CDK2 to inhibit cell proliferation and viability, and upregulated cleaved caspase-3 and Bax to induce apoptotic cell death in DS-BC cells, instead of DR-BC cells, indicating that the DR-BC cells were successfully established, which were resistant to Dox stimulation. The above results were supported by the previous studies [32, 33].

Recent data suggested that the protective autophagy could be initiated by continuously exposing to chemotherapeutic drugs in multiple cancers [17-20], and researchers noticed that activation of autophagy flux contributed to Dox-resistance in BC [21, 22], which were validated by our experiments. Specifically, the DS-BC and CR-BC cells were subjected to high-dose Dox stimulation, and we found that Dox stimulation specifically increased LC3B-II/I ratio, decreased p62 expression levels and facilitated autophagosomes formation in DR-BC cells, instead of DS-BC cells, suggesting that autophagy was activated in DR-BC cells under Dox pressure. Given the fact that autophagy protected cells from death [13, 14], we next explored whether autophagy was pivotal for regulating Dox-resistance in $B C$ cells by utilizing its inhibitor 3-methyladenine (3-MA) [23]. As expected, the results showed that 3-MA inhibited autophagy in DR-BC cells treated with Dox. Next, by performing further experiments, we evidenced that inhibition of autophagy by 3-MA inhibited cell proliferation, while promoted cell death in Dox-treated DR-BC cells, 
indicating that autophagy blockage increased Dox-sensitivity in DR-BC cells, which were partly supported by the previous data [21, 22].

AMP-activated protein kinase (AMPK)-Unc-51-like kinase 1 (ULK1) pathway involved in regulating Doxresistance in $B C$, and activation of AMPK-ULK1 pathway contributed autophagy [21]. Based on the above information, we validated that inhibition of AMPK-ULK1 pathway mediated protective autophagy increased Dox-sensitivity in DR-BC cells. Mechanistically, we noticed that high-dose Dox specifically increased the expression levels of phosphorylated AMPK (p-AMPK), p-ULK1 (555) and p-ULK1 (757) to activate AMPK-ULK1 signal pathway in DR-BC cells, instead of DS-BC cells, suggesting that AMPK-ULK1 pathway was susceptible to be activated by Dox in DR-BC cells. Next, we used the inhibitors for AMPK (compound C) and ULK1 (SBI-0206965) to block AMPK-ULK1 pathway, and the results showed that both compound $\mathrm{C}$ and SBI-0206965 reversed Dox-induced autophagy in DR-BC cells, suggesting that Dox activated autophagy in a AMPK-ULK1 pathway dependent manner, which were supported by the previous work [21]. Finally, we evidenced that inhibition of AMPK-ULK1 pathway triggered apoptotic cell death to enhance the cytotoxic effects of high-dose Dox on DR-BC cells, indicating that targeting AMPK-ULK1 pathway was effective to reduce Dox-resistance in DR-BC cells.

\section{Conclusions}

Taken together, the present study validated that inhibition of AMPK-ULK1 mediated protective autophagy was effective to increase Dox-sensitivity in BC cells in vitro, which provided alternative treatment strategies for BC treatment in clinic. However, the xenograft mice models and patients clinical specimens are still needed in our future work to verify the cellular results in vivo.

\section{Abbreviations}

BC: Breast cancer

CCK-8: Cell counting kit-8

EM: Electronic microscope

DS-BC: Doxorubicin-sensitive BC

DR-BC: Doxorubicin-resistant BC

3-MA: 3-methyladenine

Dox: Doxorubicin

NSCLC: Non-small cell lung cancer

HCC: Hepatocellular carcinoma 
LC3: Microtubule-associated protein light chain 3

AMPK-ULK1: AMP-activated protein kinase-Unc-51-like kinase 1

\section{Declarations}

\section{Ethics approval and consent to participate}

Not applicable.

\section{Consent for publication}

All the co-authors agreed to publish the final version of the manuscript.

\section{Availability of data and materials}

All the data in supportive of this work had been included in the manuscript, and the original raw data was accessible from the corresponding author with reasonable request.

\section{Conflicts of interest}

Not applicable.

\section{Funding}

This study was financially supported by Science and Technology Research Project of Heilongjiang Education Department (No. 10541144 and No. 12531275)

\section{Authors' contributions}

Dr. Libo Yu was responsible for the conception and design of this manuscript, and Dr. Libo Yu also drafted the first version of the paper. Dr. Qingtao Shi and Dr. Yan Jin conducted part of the experiments, and proofread the manuscript. Dr. Zhixin Liu and Jiaxin Li collected and analyze the data, and they also visualize the data. Dr. Wenzhou Sun was responsible for the guidance and submission of this manuscript, and Dr. Wenzhou acquired finance to support this work.

\section{Acknowledgements}

Not applicable.

\section{References}

1. Castaneda, S.A. and J. Strasser, Updates in the Treatment of Breast Cancer with Radiotherapy. Surg Oncol Clin N Am, 2017. 26(3): p. 371-382. 
2. Curigliano, G., et al., De-escalating and escalating treatments for early-stage breast cancer: the St. Gallen International Expert Consensus Conference on the Primary Therapy of Early Breast Cancer 2017. Ann Oncol, 2017. 28(8): p. 1700-1712.

3. Wang, Y.F., et al., MicroRNA-608 Promotes Apoptosis in Non-Small Cell Lung Cancer Cells Treated With Doxorubicin Through the Inhibition of TFAP4. Front Genet, 2019. 10: p. 809.

4. Jin, X., J. Zhou, Z. Zhang, and H. Lv, Doxorubicin combined with betulinic acid or lonidamine in RGD ligand-targeted pH-sensitive micellar system for ovarian cancer treatment. Int J Pharm, 2019. 571: p. 118751.

5. Wang, J., et al., Enhanced doxorubicin delivery to hepatocellular carcinoma cells via CD147 antibodyconjugated immunoliposomes. Nanomedicine, 2018. 14(6): p. 1949-1961.

6. Gao, X., Y. Wu, L. Qiao, and X. Feng, SENP2 suppresses NF-KB activation and sensitizes breast cancer cells to doxorubicin. Eur J Pharmacol, 2019. 854: p. 179-186.

7. Wei, Y., et al., Nicotinamide Overcomes Doxorubicin Resistance of Breast Cancer Cells through Deregulating SIRT1/Akt Pathway. Anticancer Agents Med Chem, 2019. 19(5): p. 687-696.

8. Zhang, Y., et al., miR-135b-5p enhances doxorubicin-sensitivity of breast cancer cells through targeting anterior gradient 2. J Exp Clin Cancer Res, 2019. 38(1): p. 26.

9. Christowitz, C., et al., Mechanisms of doxorubicin-induced drug resistance and drug resistant tumour growth in a murine breast tumour model. BMC Cancer, 2019. 19(1): p. 757.

10. Tormo, E., et al., The miRNA-449 family mediates doxorubicin resistance in triple-negative breast cancer by regulating cell cycle factors. Sci Rep, 2019. 9(1): p. 5316.

11. Lovitt, C.J., T.B. Shelper, and V.M. Avery, Doxorubicin resistance in breast cancer cells is mediated by extracellular matrix proteins. BMC Cancer, 2018. 18(1): p. 41.

12. Wu, J., H. Zhu, J. Wu, W. Chen, and X. Guan, Inhibition of N-acetyltransferase 10 using remodelin attenuates doxorubicin resistance by reversing the epithelial-mesenchymal transition in breast cancer. Am J Transl Res, 2018. 10(1): p. 256-264.

13. Cheng, Y., et al., MiRNA-224-5p inhibits autophagy in breast cancer cells via targeting Smad4. Biochem Biophys Res Commun, 2018. 506(4): p. 793-798.

14. Deng, S., et al., Targeting autophagy using natural compounds for cancer prevention and therapy. Cancer, 2019. 125(8): p. 1228-1246.

15. Chu, C.W., et al., Thioridazine Enhances P62-Mediated Autophagy and Apoptosis Through Wnt/ $\beta$ Catenin Signaling Pathway in Glioma Cells. Int J Mol Sci, 2019. 20(3).

16. Li, W., et al., Immunofluorescence Staining Protocols for Major Autophagy Proteins Including LC3, P62, and ULK1 in Mammalian Cells in Response to Normoxia and Hypoxia. Methods Mol Biol, 2019. 1854: p. 175-185.

17. Gao, J. and W. Wang, Knockdown of galectin-1 facilitated cisplatin sensitivity by inhibiting autophagy in neuroblastoma cells. Chem Biol Interact, 2019. 297: p. 50-56. 
18. Liu, F., et al., LncRNA NEAT1 knockdown attenuates autophagy to elevate 5-FU sensitivity in colorectal cancer via targeting miR-34a. Cancer Med, 2020. 9(3): p. 1079-1091.

19. Chen, H., et al., Danthron suppresses autophagy and sensitizes pancreatic cancer cells to doxorubicin. Toxicol In Vitro, 2019. 54: p. 345-353.

20. Zhou, Y., et al., miR-223 overexpression inhibits doxorubicin-induced autophagy by targeting FOXO3a and reverses chemoresistance in hepatocellular carcinoma cells. Cell Death Dis, 2019. 10(11): p. 843.

21. Liu, Z., et al., Resistin confers resistance to doxorubicin-induced apoptosis in human breast cancer cells through autophagy induction. Am J Cancer Res, 2017. 7(3): p. 574-583.

22. Liang, L., et al., MiR-142-3p enhances chemosensitivity of breast cancer cells and inhibits autophagy by targeting HMGB1. Acta Pharm Sin B, 2020. 10(6): p. 1036-1046.

23. Liu, H., et al., Autophagy inhibitor 3-methyladenine alleviates overload-exercise-induced cardiac injury in rats. Acta Pharmacol Sin, 2017. 38(7): p. 990-997.

24. Jia, J., et al., Galectins control MTOR and AMPK in response to lysosomal damage to induce autophagy. Autophagy, 2019. 15(1): p. 169-171.

25. Vargas, J.N.S., et al., Spatiotemporal Control of ULK1 Activation by NDP52 and TBK1 during Selective Autophagy. Mol Cell, 2019. 74(2): p. 347-362.e6.

26. Xie, X., et al., Downregulation of G2/mitotic-specific cyclinB1 triggers autophagy via AMPK-ULK1dependent signal pathway in nasopharyngeal carcinoma cells. Cell Death Dis, 2019. 10(2): p. 94.

27. Yu, H., et al., Nicotinamide N-methyltransferase inhibits autophagy induced by oxidative stress through suppressing the AMPK pathway in breast cancer cells. Cancer Cell Int, 2020. 20: p. 191.

28. Morsch, A., et al., Cigarette smoke exposure induces ROS-mediated autophagy by regulating sestrin, AMPK, and mTOR level in mice. Redox Rep, 2019. 24(1): p. 27-33.

29. Che, L., et al., Loss of BRUCE reduces cellular energy level and induces autophagy by driving activation of the AMPK-ULK1 autophagic initiating axis. PLoS One, 2019. 14(5): p. e0216553.

30. Duan, G., et al., Increased Autophagy Levels Mediate Cisplatin Resistance in Cisplatin-Resistant Cells While Also Rendering Them Vulnerable to Autophagy Induction. Biomed Res Int, 2018. 2018: p. 1736738.

31. Teng, J.F., et al., Polyphyllin VI, a saponin from Trillium tschonoskii Maxim. induces apoptotic and autophagic cell death via the ROS triggered mTOR signaling pathway in non-small cell lung cancer. Pharmacol Res, 2019. 147: p. 104396.

32. Liang, Y., et al., circKDM4C suppresses tumor progression and attenuates doxorubicin resistance by regulating miR-548p/PBLD axis in breast cancer. Oncogene, 2019. 38(42): p. 6850-6866.

33. Huang, J.F., et al., Overexpression of ABCB4 contributes to acquired doxorubicin resistance in breast cancer cells in vitro. Cancer Chemother Pharmacol, 2018. 82(2): p. 199-210.

34. Qiao, Z., et al., A Novel Specific Anti-CD73 Antibody Inhibits Triple-Negative Breast Cancer Cell Motility by Regulating Autophagy. Int J Mol Sci, 2019. 20(5). 
35. Wang, N., et al., XIAOPI formula promotes breast cancer chemosensitivity via inhibiting CXCL1/HMGB1-mediated autophagy. Biomed Pharmacother, 2019. 120: p. 109519.

36. Wei, T., X. Xiaojun, and C. Peilong, Magnoflorine improves sensitivity to doxorubicin (DOX) of breast cancer cells via inducing apoptosis and autophagy through AKT/mTOR and p38 signaling pathways. Biomed Pharmacother, 2020. 121: p. 109139.

37. Liu, J., et al., Blocking AMPK/ULK1-dependent autophagy promoted apoptosis and suppressed colon cancer growth. Cancer Cell Int, 2019. 19: p. 336.

\section{Figures}
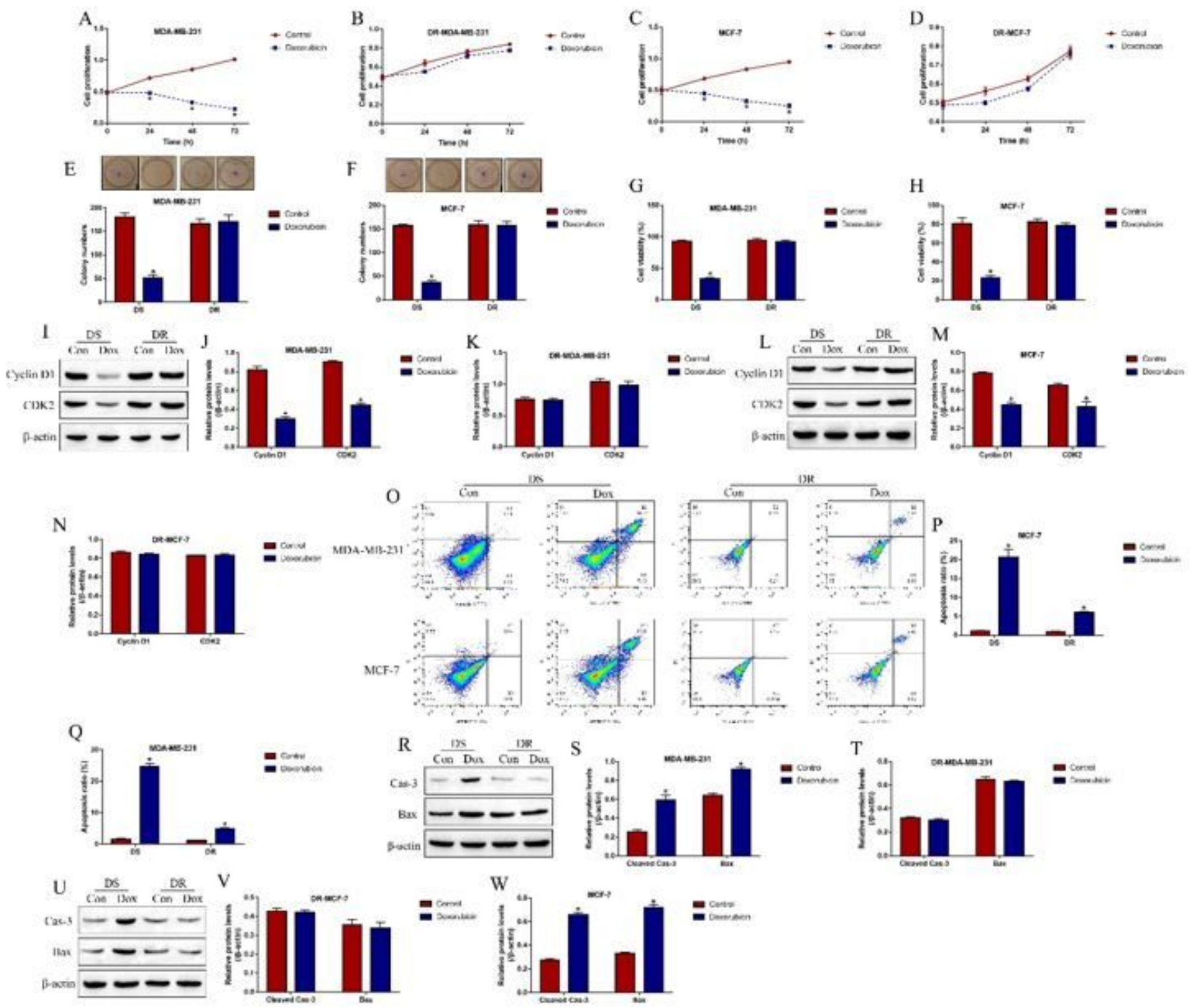

Figure 1 
Induction of DR-BC cells from their corresponding parental DS-BC cells through continuous low-dose doxorubicin pressure in a step-wise manner. (A-D) CCK-8 assay was used to determine cell proliferation abilities. (E, F) Colony formation assay was conducted to examine the colony formation abilities in $B C$ cells. $(G, H)$ Trypan blue staining assay was employed to determine cell viability. (I-N) The expression levels of proliferation associated biomarkers (Cyclin D1 and CDK2) were examined by using Western Blot analysis. (O-Q) Cell apoptosis was examined by using the Annexin V-FITC/PI double staining method. (RW) Western Blot was conducted to examine the expression levels of apoptosis associated markers (cleaved caspase-3 and Bax) in BC cells. Each experiment repeated at least 3 times, and ${ }^{*} \mathrm{P}<0.05$ was regarded as statistical significance.
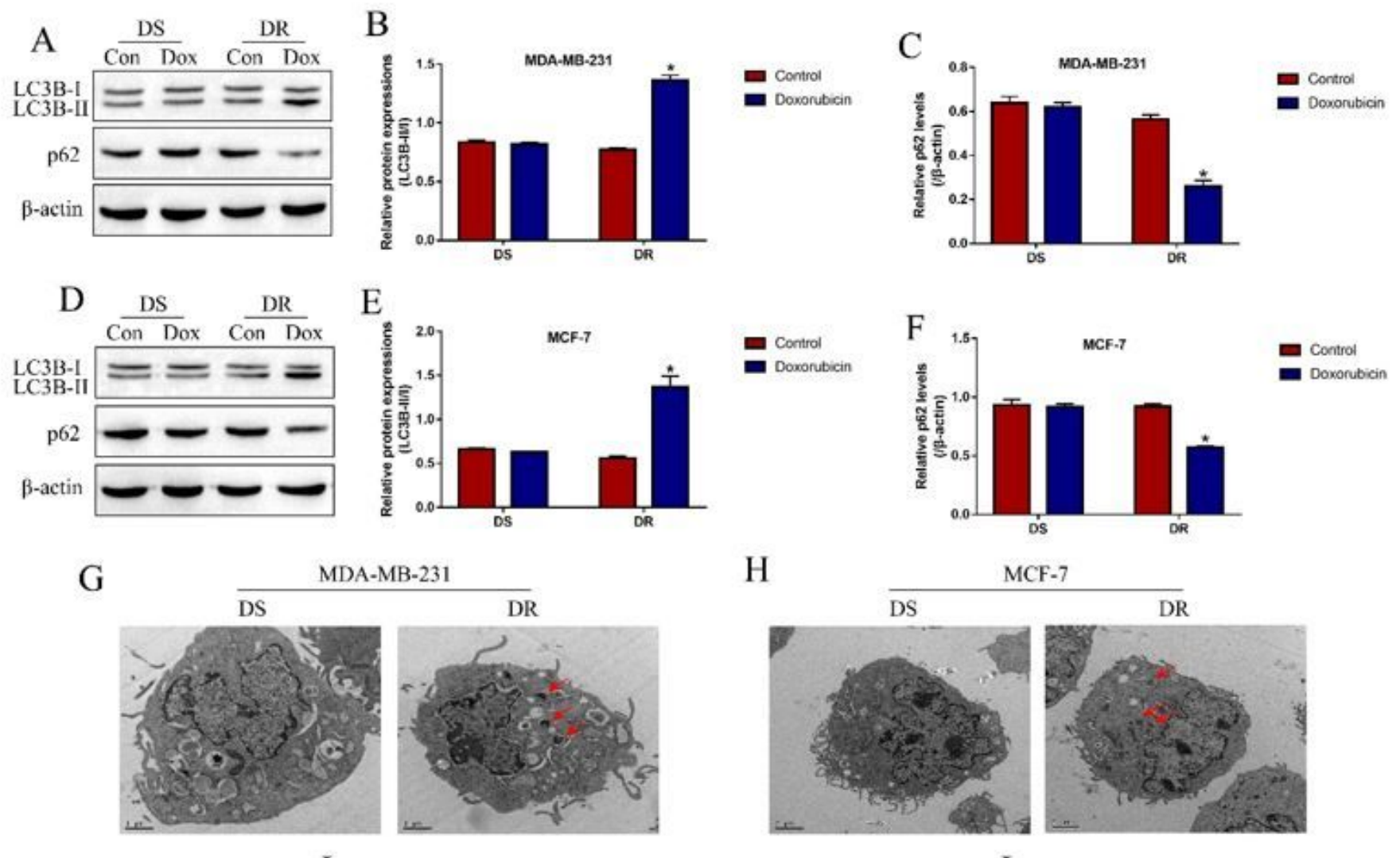

$\mathrm{H}$
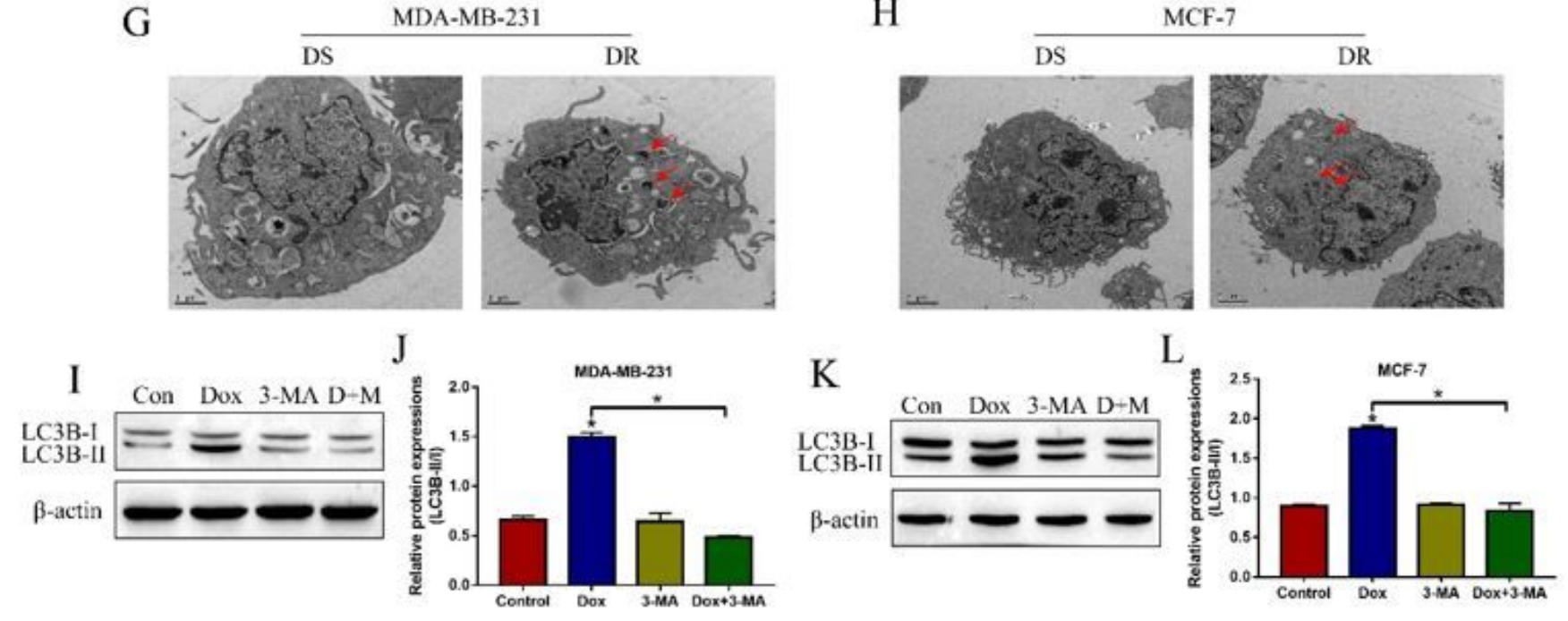

Figure 2

High-dose doxorubicin specifically activated autophagy flux in DR-BC cells, instead of their parental DS$B C$ cells. (A-F) By conducting Western Blot analysis, we found that doxorubicin specifically increased LC3B-II/I ratio, and decreased p62 expression levels in DR-BC cells. $(G, H)$ Electronic microscope (EM) was used to observe the autophagosomes (indicated by the red arrows), and the magnificence of the images 
were $5000 \times$. (I-L) The relative levels of LC3B-II/I ratio was determined by Western Blot analysis in DR-BC cells. Each experiment repeated at least 3 times, and ${ }^{*} \mathrm{P}<0.05$ was regarded as statistical significance.
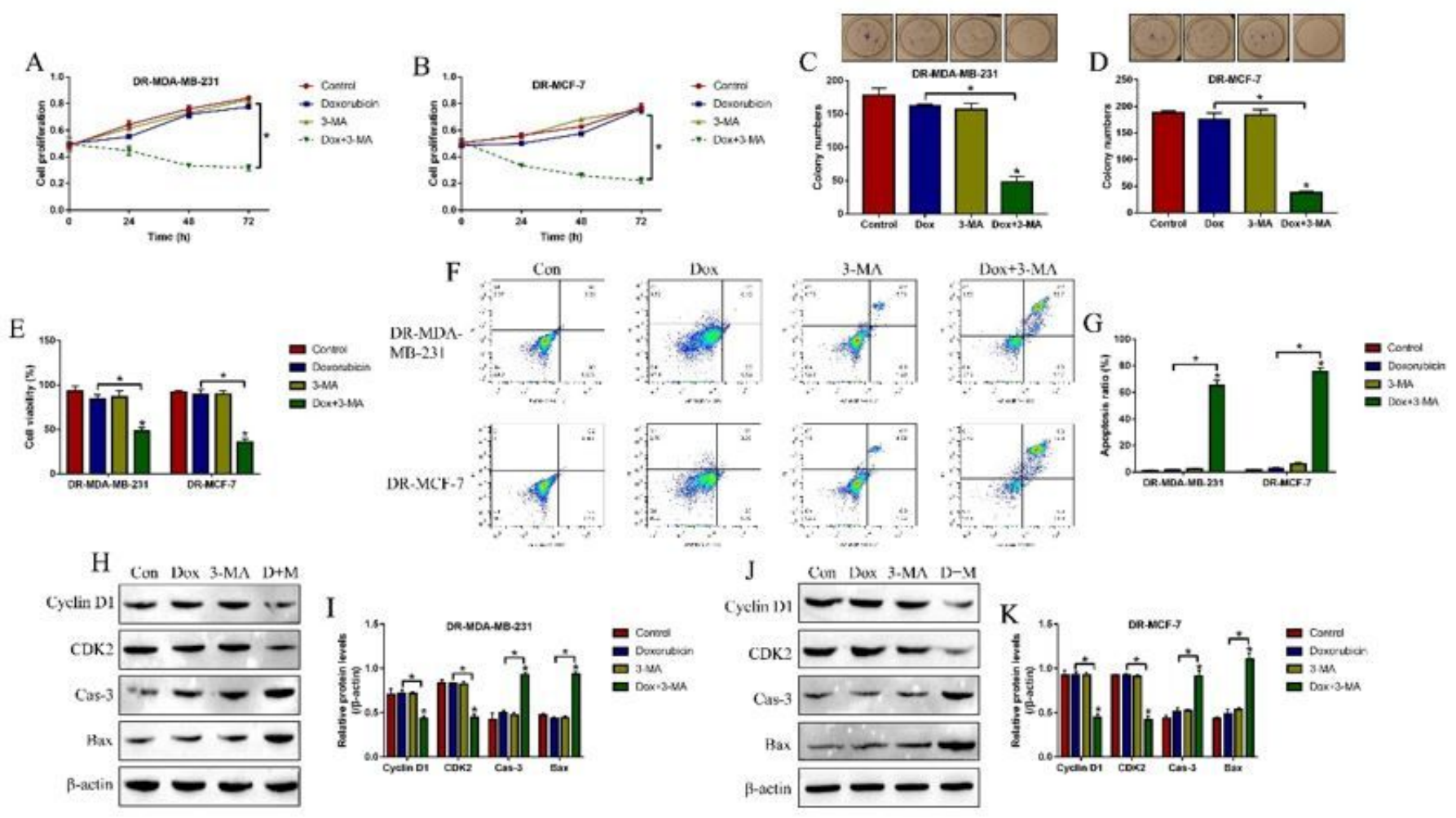

\section{Figure 3}

Inhibition of autophagy by 3-MA aggravated the promoting effects of high-dose doxorubicin-induced cell death in DR-BC cells. (A, B) Cell proliferation was evaluated by CCK-8 assay. (C, D) Colonies formation abilities were examined by colony formation assay. (E) Trypan blue staining assay was performed to determine cell viability. $(F, G)$ Cell apoptosis was determined by using the Annexin V-FITC/PI double staining method. (H-K) The expression levels of Cyclin D1, CDK2, cleaved caspase-3 and Bax were examined by using the Western Blot analysis. Each experiment repeated at least 3 times, and ${ }^{*} \mathrm{P}<0.05$ was regarded as statistical significance. 

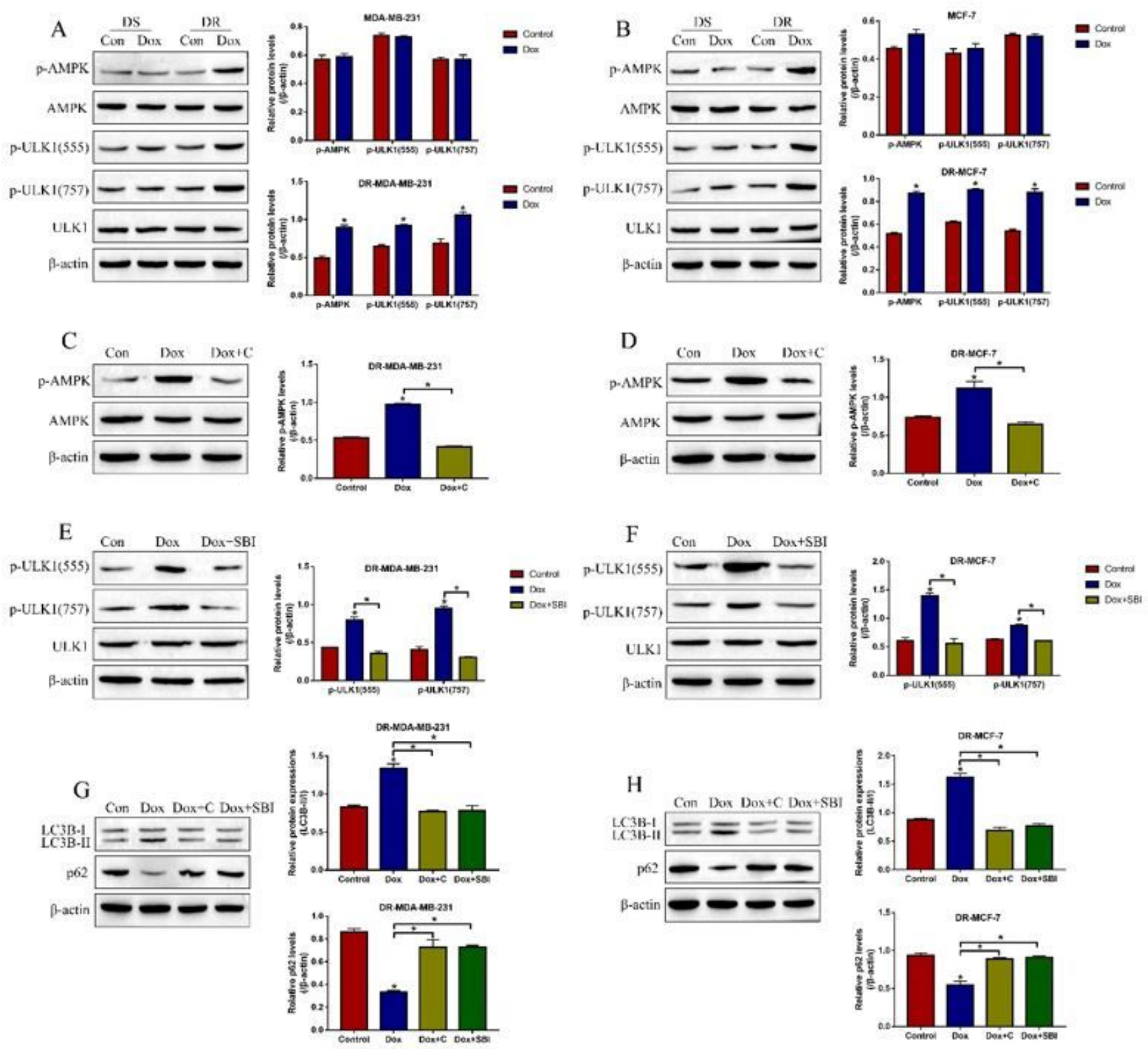

\section{Figure 4}

High-dose doxorubicin activated autophagy flux in DR-BC cells through regulating AMPK-ULK1 pathway, examined by Western Blot analysis. (A, B) High-dose doxorubicin specifically increased the expression levels of p-AMPLK and p-ULK1 to activate AMPK-ULK1 pathway in DR-BC cells, instead of DS-BC cells. The AMPK-ULK1 pathway was successfully blocked by its inhibitor, (C, D) compound C and (E, F) SBI0206965. (G, H) Blockage of AMPK-ULK1 pathway decreased LC3B-II/I ratio, and increased p62 expression levels to reverse doxorubicin induced autophagy in DR-BC cells. Each experiment repeated at least 3 times, and $* P<0.05$ was regarded as statistical significance. 

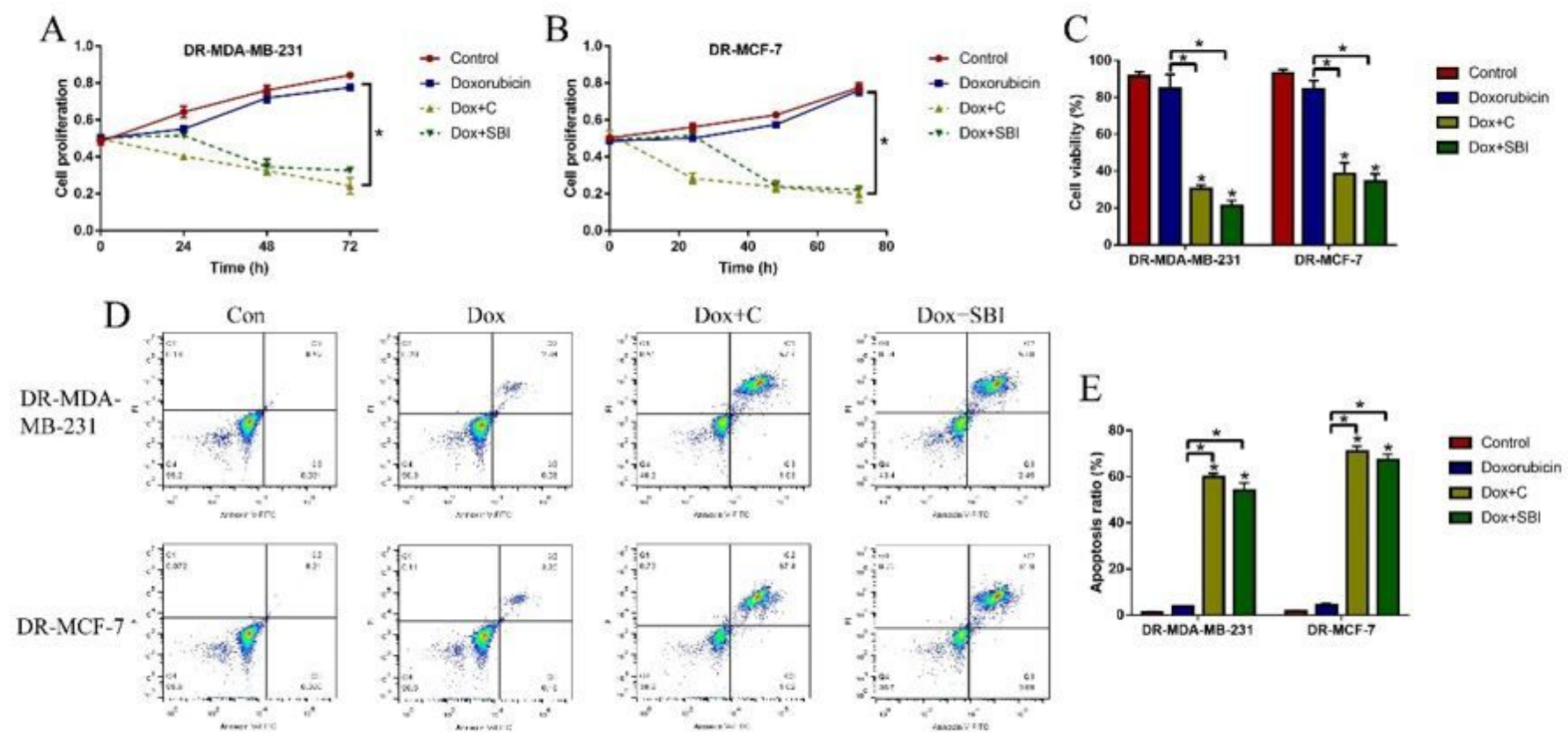

\section{Figure 5}

Inhibition of AMPK-ULK1 pathway aggravated the promoting effects of high-dose doxorubicin-induced cell death in DR-BC cells. (A, B) CCK-8 assay results indicated that blockage of AMPK-ULK1 pathway by compound $C$ and SBI-0206965 inhibited cell proliferation in DR-BC cells treated with high-dose doxorubicin. (C) Trypan blue staining assay was used to detect cell viability. (D, E) Cell apoptosis ratio was measured by using the Annexin V-FITC/PI double staining assay. Each experiment repeated at least 3 times, and $* P<0.05$ was regarded as statistical significance. 